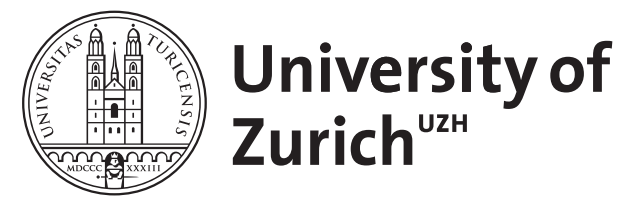

\title{
Güter, Tugenden, Pflichten
}

Fischer, Johannes

\begin{abstract}
Ist es Aufgabe der Medizinethik und medizinethischer Kommissionen, moralische Urteile von der Art zu fällen, dass eine Handlung oder Praktik, wie der assistierte Suizid, moralisch richtig oder legitim ist? Der folgende Beitrag argumentiert dafür, dass sich die Medizinethik solcher Urteile enthalten sollte. Seine These ist, dass die Aufgabe der Medizinethik nicht in moralischen Bewertungen, sondern in der Reflexion auf diejenigen Güter, Tugenden und Pflichten besteht, die bei einer solchen Handlung oder Praktik auf dem Spiel stehen. In diesem Sinne übt er Kritik an einer verbreiteten Auffassung von Moral und Ethik
\end{abstract}

DOI: https://doi.org/10.1007/s00481-006-0423-0

Other titles: Goods, virtues, duties

Posted at the Zurich Open Repository and Archive, University of Zurich

ZORA URL: https://doi.org/10.5167/uzh-156150

Journal Article

Published Version

Originally published at:

Fischer, Johannes (2006). Güter, Tugenden, Pflichten. Ethik in der Medizin, 18(2):148-163.

DOI: https://doi.org/10.1007/s00481-006-0423-0 
Johannes Fischer · Zürich

\section{Güter, Tugenden, Pflichten}

\author{
Zum sittlichen Fundament der Medizinethik
}

In einer eidgenössischen Ethikkommission für den Bereich der Humanmedizin wurde im Zusammenhang mit der Debatte über den assistierten Suizid eine Arbeitsgruppe eingesetzt, die ein Thesenpapier zur Frage der rechtlichen Regelung der Suizidbeihilfe in der Schweiz erarbeiten sollte. Diese ist nach geltender Rechtslage straffrei, sofern sie nicht aus eigennützigen Motiven erfolgt, aber es existieren keine darüber hinausgehenden Bestimmungen, etwa hinsichtlich des assistierten Suizids bei psychisch Kranken, Kindern und Jugendlichen. Ob es diesbezüglich rechtlichen Regelungsbedarf gibt, ist politisch umstritten.

Die erste These des Papiers jener Arbeitsgruppe lautete: Beihilfe zum Suizid ist in bestimmten Fällen moralisch legitim. An dieser These entzündete sich eine Kontroverse über Verständnis und Aufgabe der Ethik und insbesondere der Medizinethik sowie über die öffentliche Rolle von Ethikkommissionen. Ist es Aufgabe der Ethik und somit auch von Ethikkommissionen, derartige Urteile über moralische Richtigkeit oder Falschheit, Legitimität oder Illegitimität abzugeben? Geht es in Fragen, wie jener des assistierten Suizids, überhaupt um den binären Kode von moralisch gut oder schlecht, richtig oder falsch, legitim oder illegitim? Und woher beziehen Ethikkommissionen die Autorität, derartige Urteile abzugeben?

An dieser Kontroverse zeigt sich ein Problem, das sich auch bei anderen medizinethischen Debatten beobachten lässt. Die These jener Arbeitsgruppe war kein Zufall.
Sie war eine nur zu verständliche Reaktion auf die moralische Verurteilung des assistierten Suizids, wie sie von anderer Seite teils mit heftiger Polemik gegen die Schweizer Praxis vorgetragen wird [7]. Es ist diese Polarisierung in der öffentlichen Debatte, die den Anschein erzeugt, als sei in solchen Fragen die moralische Bewertung der Drehund Angelpunkt der ethischen Beurteilung. Ob der assistierte Suizid zuzulassen ist oder nicht, scheint dann davon abzuhängen, ob er moralisch richtig oder falsch, legitim oder verwerflich ist. Für Verwirrung sorgt dabei nicht zuletzt ein verbreitetes und auch in Lehrbüchern zu findendes Verständnis von Ethik; danach besteht deren genuine Aufgabe im Aufstellen und im Begründen derartiger moralischer Urteile.

Doch stimmt das? Absicht dieses Beitrags ist es zu verdeutlichen, dass die Moralisierung solcher Themen von den eigentlich durch die Medizinethik zu klärenden Fragen gerade ablenkt, sind diese doch von völlig anderer Art als moralische Fragen im Sinne derartiger Urteile. Es besteht hier eine Diskrepanz zwischen der Art und Weise, wie die ethische Aufgabe weithin verstanden wird, und der Art und Weise, wie die medizinethische Urteilsbildung sich tatsächlich vollzieht. Die These der folgenden Überlegungen ist, dass sich diese auf einer Ebene jenseits der Moral vollzieht, die durch die Orientierung an Gütern, Tugenden und Pflichten konstituiert wird. Das bedeutet, dass wir in einer ethischen Frage, wie der des assistierten Suizids, zu Urteilen und Entscheiden ge- 
langen können, ohne moralische Urteile über „richtig“ oder „falsch“, „legitim“ oder „verwerflich“ fällen zu müssen. Es geht dabei nicht zuletzt um eine ethische Kultur, die an der Klärung von Sachproblemen orientiert und nicht in moralischen Polarisierungen gefangen ist.

\section{Ethik und Moral}

Wie angedeutet, resultiert ein Teil der Unklarheit bezüglich der Aufgabe ethischer Stellungnahmen aus unterschiedlichen Auffassungen zu Moral und Ethik. Daher soll zunächst das geläufige Verständnis dieser Begriffe genauer betrachtet werden.

Allgemein wird Ethik als das philosophische Nachdenken über Moral bestimmt (vgl. [5], S. 2). Klärungsbedürftig an dieser Bestimmung ist erstens, was genau unter Moral zu verstehen ist, und zweitens, was es heißt, philosophisch über Moral nachzudenken.

Achtet man darauf, wie das Wort „Moral“" gebraucht wird, dann zeigt sich eine Mehrdeutigkeit. Es lässt sich zwischen einer engen und einer weiten Bedeutung unterscheiden. Die enge Bedeutung begegnet uns in Ausdrücken, wie „moralisch gut“, „moralisch schlecht“, „moralisch richtig“, "moralisch falsch“, „moralisch legitim“, „moralisch illegitim“ "usw., die ein Moment der Billigung oder Missbilligung enthalten (vgl. [3], S. 13). Mit ihnen verständigen sich die Mitglieder einer „moral community“ darüber, welche Handlungen und Verhaltensweisen unter ihnen geachtet oder geächtet sein sollen. Urteile über moralische Richtigkeit erheben dabei einen Anspruch auf allgemeine Anerkennung dieser Richtigkeit. So tut es auch das Urteil „Suizidbeihilfe ist in bestimmten Fällen moralisch legitim"." Ein solcher Anspruch verlangt von der Moral community, entgegengesetzten Einstellungen und Handlungen die Anerkennung als moralisch richtig oder legitim zu verweigern. Denn aus logischen Gründen kann sie nicht beides zugleich anerkennen. Insofern haben derartige Ur- teile einen tendenziell konfrontativen Charakter, und moralische Kontroversen drehen sich um eben solche Urteile.

Unter diesem engen Verständnis von Moral lässt sich keineswegs alles subsumieren, worüber die Ethik in ihrer Geschichte seit der griechischen Aufklärung nachgedacht hat. So fällt etwa die aristotelische Güterund Strebensethik aus diesem Verständnis heraus. Wenn zu Beginn der nikomachischen Ethik deren Grundperspektive mit der Feststellung eingeführt wird, dass „jedes praktische Können und jede wissenschaftliche Untersuchung, ebenso alles Handeln und Wählen... nach einem Gut" strebt, weshalb „Gut“ als „das Ziel, zu dem alles strebt" $\mathrm{zu}$ bestimmen ist ([1], S. 6), dann meint das Wort „Gut“ersichtlich etwas anderes als „moralisch gut“. Der Begriff des Gutes steht bei Aristoteles in Beziehung zur menschlichen Natur, die den Menschen dahingehend disponiert hat, dass er nach Gütern, wie Gesundheit, Reichtum oder Glückseligkeit, strebt, und nicht in Beziehung zu einer Moral community, die sich über das Richtige und Falsche verständigt. Begreift man also gemäß der oben gegebenen Bestimmung von „Ethik“ auch die aristotelische Ethik als ein Nachdenken über Moral, dann fasst man den Begriff Moral sehr viel weiter, als dies beim engen Verständnis der Fall ist. In diesem weiten Verständnis umfasst der Begriff alles, was seit den Anfängen der Ethik Gegenstand ethischer Reflexion gewesen ist.

Es kennzeichnet die Ethik der Moderne, dass hier das enge Verständnis von Moral

\footnotetext{
${ }^{1}$ Es ist innerhalb der Ethik umstritten, ob es sich bei einer solchen Aussage formal tatsächlich um ein Urteil handelt, mit dem - wie dies generell für Urteile gilt ein Anspruch auf allgemeine Anerkennung seiner Wahrheit oder Geltung erhoben wird. Wie immer es sich damit verhalten mag: Deutlich ist, dass die Aussage "Suizidbeihilfe ist in bestimmten Fällen moralisch legitim" ihrem Inhalt nach einen Anspruch auf allgemeine Anerkennung der moralischen Legitimität oder Richtigkeit des assistierten Suizids erhebt. Das ist von einem Anspruch auf Wahrheit zu unterscheiden. Es geht nicht um die Anerkennung der Geltung eines Urteils, sondern um die Anerkennung der Geltung einer Norm für das Verhalten der Mitglieder der Moral community.
} 
zur Dominanz gelangt ist. Nach den verheerenden Erfahrungen der Religionskriege und dem Wegfall der legitimierenden Funktion religiöser Weltbilder hat sich die Gesellschaft der Moderne einerseits als Moral community und andererseits als Rechtsgemeinschaft konstituiert, die sich die Regeln ihres Zusammenlebens selber gibt, indem sie sich darüber verständigt, welche Handlungen moralisch zu billigen oder zu missbilligen und rechtlich zulässig oder unter Sanktion zu stellen sind, kurz: was in ihr moralisch und rechtlich als verbindlich gelten soll. Die moralische Bewertung im Sinne des engen Verständnisses von Moral wird damit zum ethischen Dreh- und Angelpunkt. Mag es auch noch andere Arten der Bewertung geben, unter denen etwas gut oder ein Gut sein kann, die entscheidende Frage ist, ob es auch moralisch gut ist, d.h. die Anerkennung der Moral community im Blick auf die Regeln ihres Zusammenlebens verdient. Auch für die ethische Beurteilung des assistierten Suizids ist in dieser Perspektive ausschlaggebend, wie dieser moralisch zu bewerten ist. Insofern liegt die These jener Arbeitsgruppe ganz auf der Linie dieses modernen Moral- und Ethikverständnisses.

\section{Güter, Tugenden und Pflichten}

Gegen diese Dominanz des engen Verständnisses von Moral lässt sich nun freilich kritisch fragen, an welchen Kriterien sich die moralische Bewertung orientiert. Sind dies nur innermoralische Kriterien im Sinne des engen Verständnisses von Moral? ${ }^{2}$ Ganz offensichtlich ist dies nicht der Fall. Es lassen sich leicht Beispiele

\footnotetext{
${ }^{2}$ In der angewandten Ethik werden diesbezüglich zwei Modelle unterschieden [2], einerseits das „fundamentalistische" Modell, wonach die Geltung und Begründung eines moralischen Urteils logisch in einer übergeordneten moralischen Regel oder einem moralischen Prinzip „fundiert" ist, und andererseits das "kohärentistische" Modell, wonach ein moralisches Urteil seine Geltung aus der "kohärenten" bzw. logisch konsistenten Einbettung in die Gesamtheit der Urteile eines moralischen Überzeugungssystems bezieht.
}

dafür beibringen, dass wir Handlungen z. B. deshalb für moralisch schlecht halten, weil sie wichtige Güter verletzen, die wir geschützt sehen möchten, und zwar Güter im Sinne des aristotelischen Begriffes. Die leibliche Unversehrtheit, die Sicherheit des Eigentums oder die Freiheit, in Fragen des eigenen Lebens selbst bestimmen $\mathrm{zu}$ können, sind solche Güter. Mit der Berufung auf Güter aber verlassen wir den Diskurs über Moral im engen Verständnis und treten in einen anderen Diskurs ein, in dem es nicht um Billigung oder Missbilligung geht, sondern um die Frage, welche Güter uns wichtig sein und wofür wir folglich Sorge tragen sollten.

Orientiert man sich an den Unterscheidungen der ethischen Tradition, dann gibt es neben der Orientierung an Gütern zwei weitere Orientierungen im Handeln, die nicht unter den engen Begriff der Moral fallen, nämlich Tugenden, wie z. B. die Haltung des Respekts, und Pflichten, wie z.B. jene, in denen Eltern sich gegenüber ihren Kindern fühlen. Gewiss kann man sagen, dass, wer respektvoll handelt, in einem moralischen Sinne gut handelt. Doch tut er, was er tut, nicht deshalb, weil es moralisch gut ist, sondern aus Respekt. Ähnliches gilt für Eltern, die sich um ihres Kindes willen und nicht, weil dies moralisch gut oder vorgeschrieben ist, in der inneren Pflicht fühlen, für ihr Kind zu sorgen. ${ }^{3}$ Die Begriffe Güter, Tugenden und (außermoralische ${ }^{4}$ ) Pflichten stehen somit für drei Modi der Orientierung im Handeln, die

\footnotetext{
${ }^{3}$ Harry Frankfurt (vgl. [10], S. 98-115) illustriert dies am Beispiel einer Mutter, die versucht ist, ihr Kind im Stich zu lassen, und die dies dann aber nicht über sich bringt. Was sie zum Bleiben nötigt, ist Frankfurt zufolge nicht die moralische Erwägung, dass das Im-Stich-Lassen des Kindes eine moralische Norm verletzt oder schlecht und verwerflich ist. Vielmehr steht sie unter einer Nötigung nichtmoralischer Art, die von Frankfurt als volitionale Nötigung interpretiert wird.

${ }^{4}$ Es wird noch darauf zurückzukommen sein, dass es natürlich auch moralische Pflichten im Sinne des engen Verständnisses von Moral gibt, in denen wir innerhalb einer Moral community stehen. Im Folgenden wird das Wort "Pflicht" in einem außermoralischen Sinn gebraucht, falls dies nicht ausdrücklich anders kenntlich gemacht wird.
} 


\section{Zusammenfassung $\cdot$ Abstract}

Ethik Med 2006 · 18:148-163

DOI 10.1007/s00481-006-0423-0

C Springer Medizin Verlag 2006

Johannes Fischer

\section{Güter, Tugenden, Pflichten - Zum sittlichen Fundament der Medizinethik}

\section{Zusammenfassung}

Ist es Aufgabe der Medizinethik und medizinethischer Kommissionen, moralische Urteile von der Art zu fällen, dass eine Handlung oder Praktik, wie der assistierte Suizid, moralisch richtig oder legitim ist? Der folgende Beitrag argumentiert dafür, dass sich die Medizinethik solcher Urteile enthalten sollte. Seine These ist, dass die Aufgabe der Medizinethik nicht in moralischen Bewertungen, sondern in der Reflexion auf dieje- nigen Güter, Tugenden und Pflichten besteht, die bei einer solchen Handlung oder Praktik auf dem Spiel stehen. In diesem Sinne übt er Kritik an einer verbreiteten Auffassung von Moral und Ethik.

\section{Schlüsselwörter}

Medizinethik · Moral · Güter · Tugenden ·

Pflichten

\section{Goods, virtues, duties - On the „sittlich“ fundaments of medical ethics}

\section{Abstract}

Definition of the problem: Is it the task of medical ethics and of medical ethics committees to pass moral judgements on whether an act or a practice such as assisted suicide is morally right or legitimate?

Arguments: The article argues for not seeing the task of medical ethics in such moral appraisals but in the reflection on the goods, virtues and duties which are at stake with respect to such an act or practice. In this sense the article criticises a widespread concept of morality and ethics.

Conclusion: Medical ethics should abstain from such judgements.

\section{Keywords}

Medical ethics - Morality · Goods - Virtues . Duties 
keine moralischen im Sinne des engen Verständnisses von Moral sind.

Wie wir Handlungen als moralisch schlecht bewerten, weil sie wichtige Güter gefährden, so bewerten wir Handlungen auch als moralisch schlecht, weil sie Tugenden, wie z.B. Respekt, vermissen lassen oder Pflichten, wie Elternpflichten, verletzen. Wenn dies aber so ist, dann kehrt sich der ethische Maßstab um. Der Dreh- und Angelpunkt der ethischen Beurteilung ist dann nicht die Frage der moralischen Richtigkeit oder Falschheit. Vielmehr hat die Beantwortung dieser Frage ihr Kriterium darin, wie eine Handlung oder Praktik unter dem Gesichtspunkt von zu schützenden Gütern, einzuhaltenden Pflichten und $\mathrm{zu}$ wahrenden Tugenden zu beurteilen ist. Wie gleich zu zeigen sein wird, ist es diese Frage, die im Zentrum der medizinethischen Reflexion steht und stehen sollte.

Um die Mehrdeutigkeit des Wortes "Moral“ auszuschließen und begriffliche Klarheit zu schaffen, soll dieses Wort im Folgenden durchgehend im Sinne der engen Bedeutung verwendet werden. Das entspricht, wie gesagt, einem heute verbreiteten Verständnis von Moral. Moralisch orientiert sich hiernach, wer sich an moralischen Bewertungen orientiert und tut, was er tut, weil es moralisch gut oder richtig ist. Für die Orientierung im Handeln an Gütern, Tugenden und Pflichten soll demgegenüber im Folgenden ein anderes Wort verwendet werden. In Anknüpfung an den aristotelischen Traditionsstrang könnte sich hierzu der Ausdruck „ethisch" anbieten. Allerdings wäre dies mit einer neuen Mehrdeutigkeit erkauft, da das Wort „Ethik“ bzw. „ethisch“ in seiner geläufigen Bedeutung die philosophische Reflexion auf die Orientierung im Handeln bezeichnet. Im Deutschen legt sich in Anknüpfung an eine bestimmte Begriffsgeschichte ein anderer Ausdruck, nämlich „sittlich“, nahe. Bezeichnete er ursprünglich einmal den Gesamtbereich dessen, womit Ethik es zu tun hat, so kam er im 19. Jahrhundert in polemischer Abgrenzung von einem bestimmten Moralver- ständnis in Gebrauch [13]. Ohne Festlegung auf historische Bedeutungsgehalte, sondern strikt im hier erläuterten Sinn soll im Folgenden zwischen moralischer und sittlicher Orientierung unterschieden werden [8].

\section{Die Bedeutung der sittlichen Orientierungsebene für die Medizinethik}

Es lässt sich nun unschwer zeigen, dass die medizinethische Reflexion sich zum wesentlichen Teil auf der sittlichen Orientierungsebene bewegt. Man mag sich dies anhand der Debatte über die Forschung an embryonalen Stammzellen in Erinnerung rufen. Auf der einen Seite ging es hier um ein Gut, nämlich um das Wohl künftiger Patienten, denen vielleicht mit neuen Therapien geholfen werden kann. Andererseits berief man sich auf die Menschenwürde des Embryos, die bestimmte Pflichten für den Umgang mit menschlichen Embryonen impliziert. Die Menschenwürde wurde dabei als etwas aufgefasst, das nicht erst durch die Moral community verliehen und zuerkannt wird, sondern vielmehr deren Verständigung über das moralisch Richtige und Falsche als Kriterium vorgegeben ist. Folglich haben auch die Pflichten, zu denen die Menschenwürde des Embryos verbindet, einen außermoralischen Status, worauf noch zurückzukommen ist. Schließlich rekurrierte man auch auf Tugenden, mit denen dem Embryo zu begegnen ist, wie etwa die Haltung des Respekts [14].

Mit alledem bewegte man sich auf einer außermoralischen Ebene. Die Kontroverse drehte sich um diese Orientierungen und die Abwägung zwischen ihnen. Kommt dem menschlichen Embryo Menschenwürde $\mathrm{zu}$, und haben wir ihm gegenüber dementsprechende Pflichten? Und wenn sie ihm zukommt: Kommt sie ihm in derselben Weise zu wie einem geborenen Menschen oder in graduell abgeschwächter Weise? Und wenn in abgeschwächter Weise: Wie ist zwischen dem Gut des Wohles künftiger Patienten und den Pflichten aufgrund der Menschenwürde des Embryos abzuwägen? 
Wie die embryonale Stammzellforschung moralisch $\mathrm{zu}$ bewerten ist, ob sie moralisch geboten oder verwerflich, zu billigen oder zu missbilligen ist, das ist offensichtlich davon abhängig, zu welchen Urteilen man auf dieser außermoralischen Ebene gelangt.

Ähnliches lässt sich für andere medizinethische Fragen zeigen. Folgt man Kennern der niederländischen Debatte über die aktive Sterbehilfe, ${ }^{5}$ dann spielt dort die Berufung auf eine Tugend eine wichtige Rolle, nämlich die Barmherzigkeit. Dem steht die Pflicht entgegen, einen Menschen nicht zu töten. Anders als in den Niederlanden wird diese Pflicht in der deutschen Diskussion von vielen Beteiligten höher bewertet.

Im Blick auf die zentrale These dieses Aufsatzes gilt es nun zu sehen, dass wir in solchen Fragen $\mathrm{zu}$ ethischen Entscheidungen gelangen können, ohne moralisch urteilen zu müssen. So kann man in der Frage des assistierten Suizids unter dem Gesichtspunkt der Fürsorge für die Suizidbeihilfe im Einzelfall eintreten, ohne ein Urteil über die moralische Legitimität des assistierten Suizids fällen zu müssen. Fürsorge bedeutet hier die Erwägung, einen zum Suizid Entschlossenen mit seiner Absicht und mit dem Risiko, dass er seinem Leben auf eine schreckliche Weise ein Ende macht, nicht allein zu lassen. Man kann sich vielmehr dieses Urteils enthalten. Sagt man aufgrund einer solchen Erwägung, dass die Suizidbeihilfe im Einzelfall „,richtig“ sein kann, dann meint man „richtig im Sinne des Fürsorgegedankens“, und das ist etwas anderes als „moralisch richtig“ oder „moralisch legitim“ im Sinne des Anspruchs auf Anerkennung einer normativen Richtigkeit seitens der Moral community. Wie es ja auch etwas anderes ist zu sagen, dass das Erstreben eines Gutes aufgrund des Gütecharakters dieses Gutes „richtig“ ist, und zu sagen, dass es „moralisch richtig“ ist.

In diesem Sinne ist zwischen sittlichen und moralischen Urteilen $\mathrm{zu}$ unterschei-

\footnotetext{
${ }^{5}$ Ich beziehe mich auf eine mündliche Mitteilung von Marcus Düwell, Utrecht.
}

den. Sittliche Urteile darüber, welche Handlungen unter dem Gesichtspunkt von Gütern, Tugenden oder Pflichten „richtig“ oder „nicht richtig“ sind, erheben qua Urteil einen Anspruch auf Wahrheit oder Geltung. Moralische Urteile über „richtig“ oder „falsch“ erheben darüber hinaus einen Anspruch auf Anerkennung dieser Richtigkeit oder Falschheit seitens der Moral community im Hinblick darauf, was unter ihren Mitgliedern als normativ verbindlich gelten soll. ${ }^{6}$ Wie ausgeführt, ist die moralische Bewertung der ethische Dreh- und Angelpunkt in der Perspektive der modernen Ethikauffassung. Das hat Folgen. Können sich doch in dieser Perspektive die Akzente in Richtung auf das Kriterium der Anerkennung seitens der Moral community mit der Konsequenz verschieben, dass die sittlichen Aspekte, die bei einer Handlung oder Praktik zu bedenken sind, über der Frage der „moral correctness" in den Hintergrund geraten. Ist sie moralisch legitim, und ist dies noch dazu durch eine dafür zuständige Ethikkommission bestätigt worden, dann ist sie ethisch abgesegnet und unbedenklich.

Es ist dies der Grund, warum die Schweizerische Akademie für medizinische Wissenschaften bei ihren Richtlinien für die Betreuung von Patienten am Lebensende [17] peinlichst darauf geachtet hat, sich jedes moralischen Urteils über den ärztlich assistierten Suizid zu enthalten. Dahinter stand die Sorge, dass mit dessen moralischer Legitimation seine sittliche Problematik verdrängt wird, und dass Ärzte sich damit der

\footnotetext{
${ }^{6}$ Vgl. dazu die Fußnote zu den Kriterien der moralischen Bewertung im Abschn. "Güter, Tugenden und Pflichten“. Mit dem Urteil, dass die Sorge für das Gut der eigenen Gesundheit, z. B. durch die Ausübung einer Sportart "richtig" ist, reklamieren wir keinen Anspruch auf allgemeine Anerkennung einer verbindlichen Verhaltensnorm. Vielmehr sagen wir etwas über die Qualität dieser Handlung gemessen an diesem Gut. Dasselbe gilt für die sittliche Beurteilung einer Handlung gemessen an einer Tugend, wie der Barmherzigkeit, z. B. wenn man sagt, dass die aktive Sterbehilfe unter dem Gesichtspunkt der Barmherzigkeit "richtig" ist. Moralische Urteile können in dem außermoralischen Bezugspunkt sittlicher Urteile ihre Begründung haben, doch sind sie ihrem Sinn nach von diesen zu unterscheiden.
} 
Verantwortung enthoben sehen, in jedem Einzelfall $\mathrm{zu}$ prüfen, wie unter sittlichen Kriterien zu entscheiden ist. Die Entscheidung, Beihilfe zum Suizid zu leisten, könnte so $\mathrm{zu}$ einer Routineentscheidung werden. Eine Feststellung wie die, dass die Beihilfe zum Suizid in bestimmten Fällen moralisch legitim ist, würde nach sich ziehen, dass diese Fälle genau spezifiziert werden müssen. Somit würde eine Checkliste entstehen. Die diesbezügliche Zurückhaltung der Akademie hat ihren Niederschlag in der Formulierung gefunden, dass die Gewissensentscheidung eines Arztes, Beihilfe zum Suizid zu leisten, zu respektieren ist. Die Respektierung einer Gewissensentscheidung ist etwas anderes als die Billigung der daraus resultierenden Handlung. Ob diese als sittlich richtig zu beurteilen ist, kann aus Sicht der Akademie nur mit Blick auf den Einzelfall ${ }^{7}$ entschieden und nicht durch Richtlinien dekretiert werden. Andererseits gibt die Akademie mit dieser Formulierung zu verstehen, dass damit zu rechnen oder zumindest nicht auszuschließen ist, dass eine solche Entscheidung sittlich richtig oder vertretbar sein kann. Und sie nimmt damit einen Arzt, der eine solche Entscheidung trifft,

\footnotetext{
${ }^{7}$ Es gibt unter Ethikern die Meinung, dass es keine wirklichen Einzelfallentscheidungen gibt, sondern dass Entscheidungen immer Regeln folgen, die qua Regel mehrere Fälle unter sich fassen. Das müsste dann auch für sittliche Entscheidungen gelten. Auch hier ergäbe sich dann eine Checkliste. Doch verkennt dieser Einwand den Charakter sittlicher Orientierungen, wie etwa der Fürsorge. Dieser Begriff lässt sich nicht in Regeln ausbuchstabieren, sondern wir assoziieren unterschiedlichste Situationen und Handlungen mit ihm, die durch Ähnlichkeit verbunden sind und die in diesem Begrif ihre sprachliche Artikulation haben. Darin ist er Begriffen, wie "tapfer" oder "grausam", vergleichbar. Die Wahrnehmung einer Situation als nach Fürsorge verlangend, folgt daher nicht dem Modell der Subsumption unter eine Regel, sondern dem Modell der Assoziation mit einem grundlegenden Verhaltensmuster, das in der Situation wiedererkannt wird (vgl. die späteren Ausführungen zur Struktur sittlicher Intuitionen). Das heißt nicht, dass sich entsprechende Handlungen in einem regelfreien Raum bewegen. Vielmehr müssen sie vor geltenden Regeln gerechtfertigt werden können (vgl. die späteren Ausführungen zur Unterscheidung zwischen "begründen“ und "rechtfertigen"). Die Akademie hat denn auch Regeln im Sinne notwendiger (nicht hinreichender) Bedingungen für den ärztlich assistierten Suizid formuliert.
}

vor moralischer Verurteilung in Schutz, geht es hier doch um anderes als um Moral.

Dieser Haltung der Akademie kommt vor dem Hintergrund der politischen Debatte in der Schweiz erhebliche Bedeutung zu. Wie die These jener Arbeitsgruppe deutlich macht, spielt hier die Frage der Moral correctness eine zentrale Rolle. Auch die Akademie sah sich gewissen Erwartungen ausgesetzt, ihre bisherige Zurückhaltung, nach der der assistierte Suizid kein Teil der ärztlichen Tätigkeit ist, aufzugeben und sich moralisch zum ärztlich assistierten Suizid zu bekennen. Dahinter stand die Auffassung, sie müsse als ethische Autorität, die für die arztethischen Richtlinien in der Schweiz zuständig ist, in dieser politisch, gesellschaftlich und unter Medizinern kontroversen Frage endlich ethisch Farbe bekennen, statt sich länger bedeckt zu halten, und, da es in der Ethik um Moral geht, eine klare moralische Position beziehen.

Die Debatte illustriert, wie sehr die sittliche und die moralische Orientierungsebene in Konkurrenz und Spannung zueinander geraten können. Das ist in der deutschen Diskussion nicht anders. Die Ereiferung über die Moral correctness von Praktiken, wie aktive Sterbehilfe oder assistierter Suizid, kann den Blick dafür trüben, wie diese Praktiken in sittlicher Hinsicht zu beurteilen sind. Dieses Spannungsverhältnis ist, wie gesagt, im ethischen Denken der Moderne angelegt, das auf die Frage der moralischen Richtigkeit fokussiert ist. Die modernen ethischen Theorien drehen sich um die Frage der Begründung dieser Art von Richtigkeit, sei es kantisch, utilitaristisch, vertragstheoretisch, diskursethisch oder auf andere Weise.

Die Unterscheidung zwischen sittlichen Urteilen und Moralurteilen ist innerhalb der Medizinethik nicht etabliert. Doch lässt sich vieles ohne sie gar nicht verstehen. Das betrifft z. B. den Status medizinethischer Richtlinien. Was sie unter dem Gesichtspunkt der Fürsorge oder des $\mathrm{Pa}-$ tientenwohls für „richtig“ erklären, wird damit nicht als „moralisch richtig“ reklamiert. Die Bestimmungen medizinethi- 
scher Richtlinien sind sittliche Urteile und keine Moralurteile.

Es ist diese Unterscheidung, mit der Weichen für das Verständnis der Aufgabe der Medizinethik insgesamt gestellt werden. Nicht zuletzt geht es hier um die Einsicht, dass die Medizinethik sich selbst schadet, wenn sie mit moralischen Ansprüchen auftritt. Lehrt doch die Erfahrung, dass es eine Illusion ist zu meinen, es würden sich in medizinethischen Fragen moralische Konsense erzielen lassen. Wird daher die Aufgabe der Medizinethik und die Rolle von medizinethischen Kommissionen öffentlich in der Weise kommuniziert, dass sie kontroverse Fragen moralisch entscheiden und entsprechende Urteile abgeben sollen, dann riskiert man angesichts unüberwindlicher moralischer Dissense permanente Enttäuschungen hinsichtlich der Leistungsfähigkeit der Medizinethik. Sie erweist sich als unfähig, das einzulösen, was sie solchermaßen als Anspruch vor sich her trägt.

Anders verhält es sich, wenn die Aufgabe dahingehend kommuniziert wird, zu analysieren und ins allgemeine Bewusstsein zu heben, was in medizinethisch kontroversen Fragen in sittlicher Hinsicht auf dem Spiel steht. Hier geht es um die Frage, für den Schutz welcher Güter, die Einhaltung welcher Pflichten und die Wahrung welcher Tugenden wir im Hinblick auf das betreffende Problem Sorge tragen sollten. Auch dabei kann es selbstverständlich Meinungsverschiedenheiten geben. Aber sie verlaufen nicht entlang dem binären Kode von moralisch gut oder schlecht, richtig oder falsch, legitim oder illegitim, sondern betreffen materiale Fragen der sittlichen Orientierung und eröffnen ein Spektrum von Möglichkeiten der eigenen sittlichen Urteilsbildung für die Adressaten entsprechender ethischer Stellungnahmen.

Selbstverständlich kann diese auch in ein moralisches Urteil überführt werden. Doch liegt das Entscheidende nicht in der Einsicht, dass die betreffende Handlung oder Praktik moralisch richtig oder moralisch falsch ist, sondern in der Erkenntnis, welche Güter, Pflichten oder Tugenden durch sie tangiert sind. Hieraus leitet sich ab, warum sie moralisch richtig oder falsch ist. Insofern strapaziert die Fokussierung auf moralische Bewertungen einen sekundären Aspekt und lenkt von den eigentlich zu klärenden Fragen ab.

All dies spricht dafür, moralische Urteile aus dem medizinethischen Diskurs ganz zu verbannen. In keinem Bereich der angewandten Ethik werden strittige Fragen öffentlich so hochmoralisch und mit solchem Empörungsgestus thematisiert wie gerade im Bereich der Medizinethik. Auch wenn die Ethik als wissenschaftliche Disziplin sich sehr zu Recht von solchem Moralismus abgrenzt, muss sie sich doch fragen lassen, ob sie ihm nicht indirekt Vorschub leistet, indem sie ethische Fragen als moralische Fragen deklariert. Insbesondere Ethikkommissionen sollten hier in Anbetracht ihrer öffentlichen Funktion und Autorität gegensteuern, indem sie sich strikt moralischer Urteile enthalten. Sie sollten stattdessen ihre Aufgabe in der Förderung der sittlichen Einsicht und Orientierungsfähigkeit sehen und das ihre dazu beitragen, den medizinethischen Diskurs zu entmoralisieren.

\section{Begrenzung der Ansprüche der Moral}

Das moralzentrierte Verständnis von Ethik hat sich freilich dem heutigen Denken tief eingeprägt. Daher soll ein Einwand genauer betrachtet werden, der sich von diesem Verständnis her aufdrängt. Braucht es nicht die Moral im Interesse verbindlicher Regeln für das gesellschaftliche Zusammenleben? Gilt dies nicht auch im Hinblick auf die Handlungen und Praktiken im Bereich der Medizin? Muss daher nicht darauf insistiert werden, dass das, was wir aufgrund außermoralischer Intuitionen bezüglich Gütern, Tugenden und Pflichten für richtig halten, nicht eo ipso auch schon moralisch richtig ist, und dass es Verbindlichkeit für unser Handeln nur beanspruchen kann, wenn es auch als moralisch richtig erwiesen werden kann? 
Dazu ist zunächst an Früheres zu erinnern. Triftig wäre die Forderung, solche Intuitionen zuerst der Prüfung auf moralische Richtigkeit $\mathrm{zu}$ unterwerfen, bevor man ihnen folgt, nur dann, wenn die Moral hinsichtlich ihrer Begründung und Geltung von diesen unabhängig wäre. Denn andernfalls hängt umgekehrt die moralische Richtigkeit von ihrer intuitiven Plausibilität ab. Ersichtlich besteht jedoch eine solche Unabhängigkeit nicht. Wie ausgeführt, halten wir Handlungen für moralisch falsch oder verwerflich, weil sie Güter gefährden, oder weil sie Tugenden oder Pflichten zuwider sind, wie jenen, zu denen der Menschenwürdegedanke verbindet. Insofern verkennt dieser Einwand das Fundierungsverhältnis von moralischer und sittlicher Orientierungsebene.

Man muss sich darüber hinaus die Konsequenzen vor Augen halten, die es hat, wenn die sittliche Orientierung im Handeln solchermaßen moralisch begründungspflichtig gemacht wird. Die Ethik wird dann mit "sophisticated questions“ der Art befrachtet, ob es moralisch richtig ist, sich um kranke Angehörige zu kümmern, für die eigenen Kinder zu sorgen oder etwas gegen die weltweite Armut zu unternehmen. Was Menschen aus sittlicher Motivation um derer willen tun, auf die ihr Tun gezielt ist, das wird ihnen solchermaßen als etwas zugemutet, das sie aufgrund der Beantwortung derartiger Fragen aus moralischen Gründen tun sollen.

Die Folge ist, dass die Ethik sich davon entfernt, wie wir uns tatsächlich im Handeln orientieren. Menschen kümmern sich um ihre kranken Angehörigen, sorgen für ihre Kinder oder engagieren sich im Kampf gegen die weltweite Armut, ohne dies von der Beantwortung derartiger moralischer Fragen abhängig zu machen. Die wenigsten dürften bereit sein, das, was ihnen solchermaßen sittlich essenziell ist, in moralischen Begründungsdiskursen zur Disposition zu stellen und von deren Ausgang abhängig $\mathrm{zu}$ machen. Inwiefern ist hier überhaupt etwas moralisch $\mathrm{zu}$ begründen? Warum moralisch sein?
Verständlich ist diese Zumutung nur vor dem Hintergrund der modernen Rolle der Moral als Instrument der Selbststeuerung einer Gesellschaft, die sich die Regeln ihres Zusammenlebens autonom gibt. Der Moraldiskurs wird damit zur zentralen Steuerungsinstanz der gesellschaftlichen Koordination der Orientierung im Handeln mit der Folge, dass alles, was es sonst noch an Orientierungsquellen gibt, sich vor dem Richterstuhl der Moral daraufhin verantworten muss, ob es auch moralisch gut oder richtig ist.

Doch mit der Einforderung solch umfassender moralischer Begründungspflichten wird eine Unterscheidung außer Acht gelassen, die für das Verständnis der Funktion der Moral als gesellschaftlicher Koordinationsinstanz der Orientierung im Handeln grundlegend ist, nämlich die Unterscheidung zwischen „begründen“ und „rechtfertigen“. Wir verlangen in unserem alltäglichen Umgang von Eltern nicht, dass sie moralisch begründen müssen, warum sie für ihre Kinder sorgen. Sie dürfen und sollen dies aus sittlicher Motivation tun. Wir verlangen dagegen sehr wohl, dass Eltern sich moralisch rechtfertigen müssen, wenn sie nicht für ihre Kinder sorgen, und zwar rechtfertigen vor der moralischen Norm, dass Eltern für ihre Kinder sorgen sollen. In diesem Sinne fungiert die Moral in unserem alltäglichen Umgang als Rechtfertigungs- und nicht als Begründungsinstanz. Das eröffnet, was die Begründung betrifft, der sittlichen Orientierung im Handeln einen großen Freiraum fernab moralischer Begründungspflichten. Nur in Fällen, in denen das Verhalten von geltenden moralischen Normen abweicht, wird es moralisch in die Pflicht genommen und die Moral als verbindlicher Grund, in einer bestimmten Weise zu handeln, in Stellung gebracht.

Was aber die moralische Rechtfertigung von Handlungen oder Unterlassungen betrifft, so ist hier zuerst derjenige in Begründungspflicht, der solche Rechtfertigungen einfordert. Er muss dartun, dass gültige moralische Normen tangiert sind, 
und damit dasjenige benennen, woraufhin eine Handlung oder Unterlassung moralisch gerechtfertigt werden muss. Moralische Rechtfertigungspflichten gibt es nicht abstrakt, sodass alles Handeln ohne Angabe dessen, woraufhin es gerechtfertigt werden soll, solchen Pflichten unterschiedslos unterworfen werden könnte, sondern immer nur konkret im Hinblick auf bestimmte moralische Normen, in Bezug auf die eine Rechtfertigungspflicht begründet werden kann. Die Unterscheidung zwischen „begründen“ und „rechtfertigen" dient, so begriffen, der Begrenzung der Ansprüche der Moral. Leider ist sie in der heutigen Moraltheorie und Ethik nicht etabliert, und in den Lehrbüchern der Ethik werden die beiden Wörter synonym gebraucht, ${ }^{8}$ obgleich sie schon umgangssprachlich unterschiedliche Bedeutung haben. Wird diese Unterscheidung aber nicht beachtet, dann wachsen die Ansprüche der Moral mit der Folge, dass die Ethik sich dem entfremdet, wie wir uns tatsächlich im Handeln orientieren, ins Grenzenlose. In Anbetracht der fundamentalen Bedeutung der sittlichen Orientierung und Motivation gerade im Bereich des ärztlichen Handelns muss nicht zuletzt die Medizinethik solch überbordenden moralischen Ansprüchen widerstehen. Sie handelt sich andernfalls erhebliche Kommunikationsprobleme mit den medizinischen Praktikern ein, reagieren doch viele von ihnen zutiefst aversiv gegenüber moralischer Bevormundung seitens der professionellen Ethik.

Auch wenn medizinethische Richtlinien etwas anderes als Moralurteile sind, so ist doch die Unterscheidung zwischen „begründen“ und „rechtfertigen “ auch für deren Verständnis grundlegend. Auch sie fungieren nicht als Begründungs-, sondern als Rechtfertigungsinstanz; vor dieser muss das ärztliche Handeln jederzeit ausgewiesen werden können. Das eröffnet dem ärztlichen Handeln den notwendigen

\footnotetext{
${ }^{8}$ Vgl. dazu etwa das Begriffsregister des Handbuchs Ethik unter dem Stichwort "Rechtfertigung" ([5], S. 574).
}

Freiraum für die sittliche Orientierung an der individuellen Person, Situation und Lebensperspektive des Patienten. Dieser ist nicht ein Fall der Anwendung einer Richtlinie, sondern steht vielmehr mit seinen individuellen Bedürfnissen und Belangen im Blick. Aber das ärztliche Handeln muss mit den medizinethischen Richtlinien konform sein. Es ist dieser Status einer Rechtfertigungsinstanz, der medizinethische Regeln von den Regeln der ärztlichen Kunst unterscheidet. Haben diese doch einen begründenden Status im Hinblick darauf, was bei einem bestimmten Krankheitszustand seitens des Arztes zu tun ist, und hier ist der Patient ein Fall der subsumptiven Anwendung solcher Regeln.

\section{Nichteliminierbarkeit und rationale Überprüfbarkeit von sittlichen Intuitionen}

Ernster zu nehmen ist ein anderer Einwand. Er macht geltend, dass sittliche Intuitionen bezüglich Gütern, Tugenden und Pflichten einer rationalen Überprüfung nicht zugänglich sind und daher als Orientierungsgrundlage für die ethische Entscheidungsfindung nicht taugen. Würde man die Ethik auf diese Grundlage stellen, dann könnte jeder sich auf seine spontanen Intuitionen berufen, ohne dass zu sehen ist, wie rationale Entscheide bei divergierenden Intuitionen möglich sein sollen. Daher gelte es gerade, die ethische Urteilsund Entscheidungsfindung von Intuitionen unabhängig zu machen und auf andere Grundlagen zu stellen. Dieser Einwand bedarf einer genaueren Betrachtung.

Man muss sich hierzu die Struktur solcher Intuitionen vergegenwärtigen. Sie reagieren nicht einfach auf das, was gewissermaßen vor Augen ist, sei es real oder in der Imagination, sondern auf etwas, das damit in Verbindung gebracht wird. Im Fall der embryonalen Stammzellforschung wird die intuitive Irritation, von der viele Menschen beunruhigt sind, nicht durch den menschlichen Embryo und das, was mit diesem gemacht wird, ausgelöst, son- 
dern durch die Vorstellung, dass dies mit einem Menschen gemacht wird. Im Fall des Behandlungsabbruchs bei einem Wachkomapatienten reagiert die Intuition nicht auf den Behandlungsabbruch, sondern darauf, dass damit die Herbeiführung des Todes eines Menschen assoziiert wird. Die intuitive Perzeption nimmt mit anderen Worten nicht einfach etwas, sondern etwas in etwas wahr, wie etwa die Erniedrigung von Menschen in den Szenen von Abu Ghraib, und die intuitive Beunruhigung macht sich an dem fest, was in etwas wahrgenommen wird. Dank dieser Struktur wird die unendliche Fülle möglicher Entitäten, Situationen und Handlungen auf bestimmte sittlich relevante Szenarien und Muster reduziert, die in real begegnenden oder in der Vorstellung imaginierten Entitäten, Situationen und Handlungen wiedererkannt werden. Anders wäre gar nicht $\mathrm{zu}$ verstehen, wie wir uns in Anbetracht dieser unendlichen Komplexität sittlich in der Welt zurechtfinden können. Diese Struktur lässt sich gleichermaßen für Güter, Tugenden und Pflichten aufzeigen. Etwas wird als Gut aufgrund von Vorstellungen bezüglich seines Nutzens, die mit ihm verbunden werden, wahrgenommen. Tugenden werden dem entgegengebracht, was mit einem Wesen oder mit einer Situation assoziiert wird, wie z. B. Hilfsbedürftigkeit, auf die mit Fürsorge reagiert wird. Analoges gilt für Pflichten.

Bei dieser Struktur setzt nun die rationale Überprüfung an. Wie die entsprechenden Debatten zeigen, ist dabei zu klären, ob das, was mit dem betreffenden Vorgang assoziiert wird, zu Recht mit ihm assoziiert wird. Ist der menschliche Embryo ein Mensch im Sinne des Menschenwürdegedankens? Handelt es sich bei einem Behandlungsabbruch bei einem irreversiblen Wachkoma um ein Herbeiführen des Todes eines Menschen? Oder sollten wir eher ein Zulassen des Todes darin sehen? Und von welchen Bedingungen ist es abhängig, ob das eine oder das andere angemessen ist [9]? Solche Klärungen können intuitive Irritationen, die durch bestimmte Handlun- gen oder Praktiken ausgelöst werden, entweder bestätigen, indem sie zeigen, dass das, was darin gesehen wird, zu Recht darin gesehen werden sollte, oder aber korrigieren und beseitigen, indem sie das Gegenteil zeigen.

Es trifft also nicht $\mathrm{zu}$, dass sittliche Intuitionen einer rationalen Überprüfung nicht zugänglich sind. Man kann die entsprechenden Debatten gerade dahingehend interpretieren, dass es in ihnen um nichts anderes geht, als intuitive Irritationen, an denen sie sich entzündet haben, einer solchen Klärung zuzuführen. Und sie bedürfen solcher Klärung. Lassen sich doch die betreffenden ethischen Fragen nicht an den Intuitionen vorbei beantworten, durch die die Menschen beunruhigt sind, etwa indem man eine ethische - z. B. utilitaristische - Theorie in Stellung bringt, die die Lösung liefern soll. Denn das klärt die intuitive Beunruhigung nicht, die für die Menschen das Problem allererst zum Problem macht, für das sie eine Antwort von der Ethik erwarten. Für sie bemisst sich die Tauglichkeit einer jeden ethischen Antwort - also auch einer ethischen Theorie - daran, ob sie ihr Problem löst und die intuitive Beunruhigung rational aufklärt. Setzt sich die Ethik darüber hinweg, dann wird sie zur öffentlichen Klärung von Fragen, wie sie durch die embryonale Stammzellforschung oder den Behandlungsabbruch bei Wachkomapatienten aufgeworfen werden, nichts beitragen.

Es gilt daher, solche Intuitionen gerade nicht aus der ethischen Urteils- und Entscheidungsfindung auszuschließen, sondern sie ethisch ernst zu nehmen. Sie liefern gewissermaßen das Material, das ethisch zu bearbeiten ist. Dass wir überhaupt über Fragen, wie jene, ob in einem Embryo ein Mensch zu sehen ist oder in einem Behandlungsabbruch bei einem Wachkoma Tötung zu sehen ist, debattieren, das verdankt sich, wie gezeigt, der assoziativen Struktur solcher Intuitionen. Woher sollte es sonst kommen? Welche Erklärung haben diejenigen dafür, die für 
den Ausschluss von Intuitionen aus dem ethischen Diskurs plädieren? ${ }^{9}$

Die ethische Aufgabe besteht hier erstens darin, sprachlich zu artikulieren und $\mathrm{zu}$ explizieren, was genau es ist, das intuitiv mit bestimmten Handlungen und Situationen in Verbindung gebracht wird und entsprechende Irritationen auslöst. Häufig ist dies alles andere als klar, wie sich an den entsprechenden Argumenten zeigt. Ist es die Verletzung der Heiligkeit menschlichen Lebens, deren sich die embryonale Stammzellforschung schuldig macht? Oder ist es die Verletzung der Menschenwürde? Ist also der Embryo als „menschliches Leben“" wahrzunehmen und zu beschreiben oder als „Mensch“ im Sinne des Menschenwürdegedankens?

Die ethische Aufgabe besteht zweitens darin, solche Explikationen dem Kohärenztest mit unseren sonstigen sittlichen Überzeugungen zu unterwerfen. So erachten wir keineswegs alles, was sich als „menschliches Leben“ klassifizieren lässt Keimzellen, eine Hautzelle, ein explantiertes Organ - als heilig, unantastbar und der verbrauchenden Forschung entzogen, und das setzt ein Fragezeichen hinter die Berufung auf die Heiligkeit menschlichen Lebens in der Embryonendebatte. Was die Beschreibung „Mensch“ betrifft, so ist unseren sittlichen Einstellungen ein kategorialer Unterschied zwischen Entitäten, die „etwas" sind, und solchen, die ,jemand“ sind, eingeschrieben, d.h. zwischen dem Organismus eines Menschen, der in die Perspektive der Biologie fällt, und der Person dieses Menschen. Das bedeutet, dass der Embryo als biologische Entität sich allenfalls als das organismische Substrat ei-

\footnotetext{
${ }^{9}$ Die Entwicklungen im Bereich der Biotechnologie und der Medizin haben die Grenzen rein moralbezogener ethischer Ansätze, wie z.B. der Diskursethik, offensichtlich werden lassen. Das zeigt sich daran, dass auch Vertreter solcher Ansätze nicht umhin können, sich angesichts als "obszön" verabscheuter Szenarien im Interesse der Begrenzung des technologisch Machbaren auf elementare Intuitionen zu berufen. Instruktiv sind diesbezüglich Jürgen Habermas' Erwägungen zur "Würde menschlichen Lebens" und zur "gattungsethischen Einbettung der Moral" $^{\prime \prime}[11]$, S. 56-80).
}

nes Menschen, nicht aber als dieser Mensch selbst verstehen lässt. Die Auffassung des Embryos als Mensch im Sinne des Menschenwürdegedankens erweist sich damit als mit unseren sonstigen sittlichen Überzeugungen inkohärent, und die Rede von der Menschenwürde des Embryos unterliegt dem Verdacht auf ein kategoriales Missverständnis. ${ }^{10}$

Gerade weil die Medizin sich in einem Bereich bewegt, der mit starken Intuitionen besetzt ist, hat die Medizinethik in vielen Fällen eine aufklärende Funktion in Bezug auf ein Bewusstsein, das intuitiv durch bestimmte Dinge irritiert ist, aber die Gründe nicht kennt, aufgrund deren es irritiert ist, und das daher auch nicht die Stichhaltigkeit dieser Gründe überprüfen kann. Die ethische Aufgabe besteht demgemäß darin, dieses Bewusstsein zu analysieren sowie die Gründe herauszufinden und $\mathrm{zu}$ formulieren, die die Irritation einfangen, und sie dem Kohärenztest zu unterwerfen. Mit einer Unterscheidung von Wilhelm Dilthey (vgl. [4]) lässt sich dabei zwischen drei Ebenen differenzieren, auf denen sich dieser Prozess vollzieht: die Ebene des intuitiven Erlebens, das auf reale oder in der Vorstellung imaginierte Szenarien reagiert, die Ebene des sprachlichen Ausdrucks oder der Artikulation des solchermaßen Erlebten sowie die Ebene des Verstehens des solchermaßen Artikulierten.

\section{Menschenwürde und Differenzierung zwischen sittlichen und moralischen Begriffen}

Eines der stärksten Argumente für den Primat der sittlichen Orientierungsebene für die Ethik ist die nicht nur in medizinethischen Debatten anzutreffende Berufung auf die Menschenwürde. Denn Menschenwürde ist ein sittlicher und kein moralischer Begriff. Sie ist, wie gesagt, nicht durch eine Moral community verliehen, die sich darüber verständigt, was für ihre Mitglieder als verbindlich gelten soll, sondern

${ }^{10} \mathrm{Vgl}$. zum Standpunkt des Autors in dieser Frage [6]. 
vielmehr deren Verständigung über das moralisch Richtige und Falsche als Kriterium vorgegeben. Im Sinne der Unterscheidung Diltheys lässt sich der Menschenwürdebegriff als sprachliche Artikulation unserer intuitiven Vorstellungen dessen verstehen, was mit einem Menschen unter keinen Umständen geschehen darf. So begriffen, bezeichnet er nicht ein moralisches Recht oder einen moralischen Anspruch, sondern etwas, aufgrund dessen moralische Rechte in Gestalt der Menschenrechte zuerkannt werden. ${ }^{11}$ Es ist in diesem sittlichen Charakter der Menschenwürde begründet, dass die Menschenrechte als moralische Rechte nicht in einem logischen Sinn aus ihr abgeleitet werden können.

Wie sich an diesem Beispiel zeigt, ist es nützlich und klärend, das ethische Vokabu-

${ }^{11}$ Auch anderen Konzeptualisierungen des Menschenwürdebegriffs liegt die Berufung auf eine Intuition zugrunde. So sieht Peter Schaber den Gehalt des Menschenwürdegedankens darin, dass ein Mensch nicht erniedrigt werden darf [15]. Unklar bleibt dabei allerdings die Zuordnung des Menschenwürdebegriffs zur sittlichen oder zur moralischen Sphäre. Einerseits wird die Verletzung der Menschenwürde von Schaber als Erniedrigung eines Menschen bestimmt; dies entspricht einer sittlichen Intuition. Andererseits wird die Menschenwürde als der moralische Anspruch oder das moralische Recht definiert, nicht erniedrigt zu werden; dies bedeutet, dass die Verletzung der Menschenwürde in der Verletzung dieses moralischen Rechtes besteht. Das ist ersichtlich zweierlei. Oder soll beides in der Weise zusammengenommen werden, dass die Erniedrigung in der Verletzung des moralischen Rechtes besteht, nicht erniedrigt zu werden - was dann aber hieße: nicht in seinem moralischen Recht verletzt zu werden, nicht erniedrigt usw. - ? Was haben wir bei den Szenen aus Abu Ghraib vor Augen: die Erniedrigung von Menschen oder die Verletzung moralischer Rechte? Fraglich ist darüber hinaus, ob es beim Gedanken der Menschenwürde tatsächlich nur um Erniedrigung geht. Wenn im Bosnienkrieg die serbische Soldateska mit Lastwagen über gefangene Muslime gefahren ist, dann ist dies zweifellos ein Fall von Verletzung der Menschenwürde. Doch soll diese wirklich nur darin gelegen haben, dass die Muslime durch diese Art der Tötung erniedrigt worden sind? Oder lag sie nicht vielmehr darin, dass sie auf eine grässliche Weise umgebracht worden sind? Muss also der Begriff der Menschenwürde nicht dahingehend, was man mit Menschen qua Menschen unter keinen Umständen macht bzw. machen darf, weiter gefasst werden? Mit dieser Frage bewegt man sich im Sinne der Unterscheidung Diltheys auf der Ebene des Verstehens, das sich seinerseits als Arbeit an der Artikulation begreifen lässt. lar daraufhin $\mathrm{zu}$ sortieren, ob es der sittlichen oder der moralischen Sphäre zuzuordnen ist. Güter, Tugenden und (außermoralische) Pflichten, zu denen der Menschenwürdegedanke gehört, konstituieren die sittliche Sphäre. Sie sind ihrer Form und Eigenart nach - nicht in ihrer konkreten Gestalt, bei der kulturelle und soziale Einflüsse eine Rolle spielen - in der menschlichen Natur angelegt, die wir bis zu einem gewissen Grad mit höher entwickelten Tieren teilen. Auch sie streben aufgrund ihrer Bedürfnisnatur nach Gütern, sind empfänglich für Haltungen, wie Zugewandtheit oder Fürsorglichkeit, wie sie auch selbst solche Haltungen gegenüber ihren Artgenossen zeigen, und reagieren instinktiv genötigt auf bestimmte Situationen, z. B. im Fall der Bedrohung ihrer Jungen.

Normen, Werte oder Rechte, insoweit sie Thema der Ethik sind, gehören demgegenüber dem Bereich der Moral zu. Sie sind Gegenstand der Verständigung der Moral community darüber, woran sich das Verhalten ihrer Mitglieder orientieren, und was für diese verbindlich sein soll. Für den Begriff der Norm liegt dies auf der Hand. Doch auch der Begriff des Wertes bezeichnet im Unterschied $\mathrm{zu}$ jenem des Gutes nichts, was in der menschlichen Naturanlage fundiert ist, sondern er ist als ethischer Begriff ein Konstrukt des ethischen Diskurses, das eine Warum-Frage beantworten soll, nämlich warum wir bestimmte Handlungen vollziehen oder bestimmte Dinge schützen bzw. schonend mit ihnen umgehen sollen. Die Antwort ist: weil dem, was sich in solchen Handlungen realisiert, ein Wert zukommt bzw. weil jene Dinge (intrinsisch) wertvoll sind. ${ }^{12}$ Der Wertbegriff steht für dasjenige, was der Grund ist, warum wir bestimmte Handlungen und Dinge vorziehen und andere hintansetzen (sollten). Als solches Konstrukt ist er philosophiegeschichtlich vergleichsweise jung (vgl. dazu [16], S. 198-234). Bei Rechten schließlich handelt es sich um An-

\footnotetext{
${ }^{12}$ Fußnote siehe Seite 161.
} 
sprüche, die die Mitglieder einer Moral community gegeneinander haben, und die sie sich sowie anderen Wesen, wie z. B. Tieren, zuerkennen. Ihnen korrespondieren Pflichten aufseiten anderer. Letzteres bedeutet, dass in Bezug auf den Pflichtbegriff zwischen sittlichen Pflichten, wie jenen, in denen sich Eltern gegenüber ihren Kindern fühlen, und moralischen Pflichten, in denen wir als Mitglieder einer Moral community stehen, differenziert werden muss.

Wie die vorstehenden Ausführungen zu der Frage verdeutlichen sollten, ob moralische Urteile ihre Begründung und Geltung rein moralimmanent ableiten oder über den Bezug auf außermoralische sittliche Orientierungen, ist davon auszugehen, dass die Moral community bei der Verständigung darüber, welche Handlungen richtig oder falsch, gut oder schlecht sind, oder welche Rechte Menschen oder Tieren zuzuerkennen sind, ihre sittlichen Orientierungen nicht einfach beiseite stellt, sondern vielmehr durch diese geleitet ist. Sie überführt in solcher Verständigung das, was ihr in sittlicher Hinsicht wesentlich ist, in für ihre Mitglieder verbindliche moralische Normen, Werte, Rechte und Pflichten. So lässt sich, wie gesagt, das moralische Postulat von Menschenrechten als durch die sittlichen Pflichten motiviert verstehen, in denen wir uns in Bezug auf

\footnotetext{
12 Dies unterscheidet den Wertbegriff als moralischen Begriff vom sittlichen Begriff der Menschenwürde. Denn wenn dieser im Sinne der Artikulation unserer intuitiven Vorstellungen davon aufzufassen ist, was einem Menschen qua Menschen angemessen oder unangemessen ist, dann kann die Menschenwürde nicht als ein solcher "Basisgrund" ([18], S. 149f.) für die Begründung des Gebots oder Verbots bestimmter Handlungen in Anspruch genommen werden. Ein Satz, wie "Weil X Menschenwürde hat, darf X nicht gefoltert werden", ist dann tautologisch. Denn der Begriff der Menschenwürde ist u.a. über die Vorstellung gebildet, dass ein Mensch nicht gefoltert werden darf. Sachlich angemessen, kann man nur sagen, dass der Ausschluss der Folter zum Gehalt des Menschenwürdegedankens gehört. Werte hingegen fungieren als solche Basisgründe, etwa im Sinne der Feststellung, dass die Natur schonend zu behandeln ist, weil sie einen intrinsischen Wert hat. Aufgrund dieses unterschiedlichen Status ist die Interpretation der Menschenwürde als intrinsischer Wert des Menschen fragwürdig.
}

den Schutz der Menschenwürde sehen, und es transformiert über die Zuerkennung von Rechten diese sittlichen Pflichten in moralische Pflichten, die für die globale Moral community verbindlich sind.

Im Blick auf das Verhältnis von moralischer und sittlicher Orientierung bleibt hinzuzufügen, dass die innerhalb einer Moral community herrschende Moral selbstverständlich Rückwirkungen auf deren sittliches Bewusstsein hat, insofern bestimmte Handlungen und Verhaltensweisen durch sie geächtet werden und dadurch einer Gewöhnung an sie vorgebeugt wird. Daher ist die Moral in Verbindung mit dem Recht eine unverzichtbare Institution im Hinblick auf die Wahrung der sittlichen Sensibilität einer sozialen Gemeinschaft. Die moralische Zuerkennung von Menschenrechten und die Statuierung entsprechender moralischer Pflichten dienen, so gesehen, der Wahrung der sittlichen Orientierung an der Menschenwürde, in der sie gleichzeitig fundiert sind.

\section{Der sittliche Sinn der Medizin}

Güter, Tugenden und Pflichten sind die drei sittlichen Grundorientierungen der Ethik und daher auch der Medizinethik. Sie strukturieren zusammen das Feld, mit dem die Ethik es jenseits der Moral zu tun hat.

Innerhalb der philosophischen und theologischen Tradition gibt es eine Debatte darüber, ob einer dieser drei Orientierungen ein sittlicher Primat gegenüber den beiden anderen zukommt. Damit ist ein Primat in dem Sinne gemeint, dass der sittliche Charakter der beiden anderen Orientierungen aus der sittlichen Vorzüglichkeit dieser Orientierung abgeleitet ist. Als ein Kandidat hierfür drängt sich die Güterorientierung auf. Pflichten und Tugenden wären dann nicht um ihrer selbst willen sittlich bedeutsam, sondern um der Realisierung bestimmter sittlich vorzüglicher Güter willen, die aus dem pflicht- und tugendgemäßen Handeln resultieren. Eine solche Vorordnung der Gütersphäre 
könnte sich gerade in medizinethischer Perspektive aufgrund des praktischen, güterbezogenen Charakters der Medizin nahe legen. Sie erscheint nicht zuletzt deshalb als attraktiv, weil sie die ethische Aufgabe eminent vereinfachen würde. Alle sittlichen Konflikte ließen sich durch Güterabwägungen lösen. Auch die Pflicht der Achtung der Menschenwürde wäre dann Güterabwägungen zugänglich. Denn wenn diese Pflicht um eines Gutes willen sittlich geboten ist, dann muss im Fall eines Konfliktes mit einem anderen Gut durch Güterabwägung geprüft werden, welches das höhere Gut ist.

Doch ersichtlich steht eine solche Vorordnung der Gütersphäre im Widerspruch zu der Art und Weise, wie wir uns tatsächlich im Hinblick auf Tugenden und Pflichten orientieren. So ist die intuitive Reaktion auf Verletzungen der Menschenwürde, wie in Abu Ghraib, in Bezug auf bestimmte Güter, die dadurch gefährdet werden, nicht konsequenzialistisch motiviert. Vielmehr reagiert sie unmittelbar auf das, was vor Augen ist. Und so erachten wir ja auch sexuelle Übergriffe in therapeutischen Beziehungen nicht nur deshalb als problematisch, weil sie ein Gut in Gestalt des Therapieziels gefährden können, sondern vielmehr deshalb, weil der Arzt damit eine sittliche (und moralische) Pflicht verletzt, nämlich die Abhängigkeit des Patienten oder der Patientin nicht auszunutzen. Güter, Pflichten und Tugenden sind von einander unabhängige, komplementäre, sich wechselseitig ergänzende Orientierungen.

Diese Komplementarität erweist sich nicht zuletzt bei der Frage nach dem sittlichen Sinn der Medizin als solcher. Es ist dies eine Frage, die innerhalb der Medizinethik eher am Rande liegt. Doch kommt ihr zentrale Bedeutung im Hinblick auf die Debatte über die Ziele der Medizin zu. Im Unterschied zu einer theoretischen Disziplin, wie der Physik, die ihre Einheit von dem Gegenstandsbereich her bezieht, mit dem sie befasst ist, bezieht die Medizin ihre Einheit von diesen Zielen her. Wissen- schaftliche Forschungen sind nur dann medizinische, wenn und insofern sie an diesen Zielen orientiert sind. Abgesehen davon, fallen sie in das Gebiet der unterschiedlichsten theoretischen Disziplinen, wie der Chemie oder der Soziologie. Als praktische Disziplin muss die Medizin daher ein eminentes Interesse daran haben, sich über diese Ziele Klarheit zu verschaffen, denn hierin ist ihre disziplinäre Identität begründet. Der Hastings-Report [12], der das Resultat eines internationalen Verständigungsprozesses über die Ziele der Medizin ist, unterscheidet aufgrund der Spezifizierung bestimmter Ziele Anwendungen medizinischen Wissens und Könnens, die genuin medizinische sind, und solche, die keine genuin medizinischen sind, aber zu den Zielen der Medizin nicht im Widerspruch stehen, ${ }^{13}$ und schließlich solche, die mit den Zielen, denen die Medizin verpflichtet ist, unvereinbar sind, wie etwa die Folter. In Zeiten einer sich rapide ausweitenden Beanspruchung medizinischen Wissens und Könnens für die unterschiedlichsten Ziele ist es eine für die Medizin essenzielle Frage, über welche Ziele sie sich als Medizin definieren will. Von ihrer eigenen Geschichte her ist die Medizin Zielen verpflichtet, die es mit einer Seite menschlicher Existenz zu tun haben, die eine sittliche Herausforderung darstellt, nämlich mit der menschlichen Angewiesenheit und Abhängigkeit im Fall von Krankheit, krankheitsbedingtem Leiden und Sterben.

Eine sittliche Herausforderung ist dies gleichermaßen unter dem Gesichtspunkt von Pflichten, die wir solchermaßen angewiesenen Menschen gegenüber haben, wie unter dem Gesichtspunkt von Tugenden, wie Zuwendung und Fürsorge, die wir ih-

\footnotetext{
${ }^{13}$ Dieser Kategorie ordnet die Schweizerische Akademie für medizinische Wissenschaften den assistierten Suizid zu, wenn sie in ihren Richtlinien für die Betreuung von Patienten am Lebensende feststellt, dass dieser kein Teil der ärztlichen Tätigkeit, sondern eine persönliche Gewissensentscheidung des Arztes ist. Dies steht freilich in Spannung dazu, dass sie gleichwohl in arztethischen Richtlinien Regeln und Bedingungen dafür formuliert. Diese Spannung resultiert aus der besonderen Schweizer Situation und Rechtslage.
} 
nen entgegenbringen, wie unter dem Gesichtspunkt von Gütern - im weitesten Sinne ihres Wohles -, für die wir um ihretwillen besorgt sein müssen. So wirken hier alle drei Modi der sittlichen Orientierung im Handeln zusammen. Die entscheidende Frage in der Debatte über die Ziele der Medizin ist, ob die Medizin von diesem sittlichen Auftrag am kranken Menschen her zu begreifen ist und ihre Ziele dementsprechend einzugrenzen sind, oder ob sie für beliebige Ziele mit der Folge, dass sie ihre disziplinäre Einheit verliert und am Ende alles Medizin ist, was Mediziner aufgrund ihrer medizinischen Kompetenzen tun, fungibel werden soll.

\section{Anschrift}

\section{Prof. Dr. theol. Johannes Fischer}

Institut für Sozialtechnik, Ethik-Zentrum,

Universität Zürich, Zollikerstr. 117,

8008 Zürich, Schweiz

E-mail: fischer@sozethik.unizh.ch

\section{Danksagung}

Zu danken habe ich Stefan Gruden für kritische Anmerkungen und Rückfragen, die mich zu Verdeutlichungen genötigt haben, sowie für die Fertigstellung des Manuskripts für den Druck. Mein Dank gilt außerdem den Mitgliedern des Ethikzentrums der Universität Zürich, mit denen ich eine Vorfassung dieses Textes und seiner zentralen These diskutieren konnte.

\section{Literatur}

1. Aristoteles (1991) Nikomachische Ethik, 6. Aufl. Übersetzt und kommentiert von Franz Dirlmeier. Wissenschaftliche Buchgesellschaft, Darmstadt

2. Badura J (2002) Kohärentismus. In: Düwell M, Hübenthal C, Werner MH (Hrsg), S 194-205

3. Birnbacher D (2003) Analytische Einführung in die Ethik. Walter de Gruyter, Berlin

4. Dilthey W (1927) Der Aufbau der geschichtlichen Welt in den Geisteswissenschaften, GS VII. Teubner, Leipzig

5. Düwell M, Hübenthal C, Werner MH (2002) Handbuch Ethik. Metzler, Stuttgart

6. Fischer J (2002) Wenn "etwas" zu "jemandem" wird. Zum Status überzähliger Embryonen. Neue Zürcher Zeitung, 10./11.08.2002

7. Fischer J (2004) Die Schweizerische Akademie der medizinischen Wissenschaften zur Suizidbeihilfe. Ethik Med 16:165-169

8. Fischer J (2005) Moralische und sittliche Orientierung. ThLZ 130: 472-488

9. Fischer J (2005) Sterben hat seine Zeit. ZThK 102: 352-370

10. Frankfurt H (2001) Über die Bedeutsamkeit des Sich-Sorgens. In: Frankfurt H (Hrsg) Freiheit und Selbstbestimmung. Suhrkamp, Frankfurt a.M.

11. Habermas J (2001) Die Zukunft der menschlichen Natur. Auf dem Weg zu einer liberalen Eugenik? Suhrkamp, Frankfurt a.M.

12. Hastings Center (1996) The goals of medicine. Setting new priorities. The Hastings Center, Briarcliff Manor, NY

13. Kersting W (1995) Art. "Sittlichkeit; Sittenlehre". In: Ritter J, Gründer K (Hrsg) Historisches Wörterbuch der Philosophie, Bd. 9. Wissenschaftliche Buchgesellschaft, Darmstadt, S 907923

14. Maio G (2002) Welchen Respekt schulden wir dem Embryo? Die embryonale Stammzellforschung in medizinethischer Perspektive. Dtsch Med Wochenschr 127: 160-163

15. Schaber $P$ (2004) Menschenwürde und Selbstachtung. Ein Vorschlag zum Verständnis der Menschenwürde. Stud philos 63: 93-106

16. Schnädelbach H (1983) Philosophie in Deutschland 1831-1933. Suhrkamp, Frankfurt a.M.

17. Schweizerische Akademie der medizinischen Wissenschaften (2004) Richtlinien für die Betreuung von Patientinnen und Patienten am Lebensende. http://www.samw.ch. Gesehen 29. Apr 2006

18. Taylor C (1996) Die Quellen des Selbst. Suhrkamp, Frankfurt a.M. 